\title{
Radiative Interaction of Electrons in a Bunch Moving in an Undulator
}

\author{
E.L. Saldin ${ }^{\text {a }}$, E.A. Schneidmiller ${ }^{\text {a }}$, M.V. Yurkov ${ }^{b}$ \\ a Automatic Systems Corporation, 443050 Samara, Russia \\ ${ }^{b}$ Joint Institute for Nuclear Research, Dubna, 141980 Moscow Region, Russia
}

\begin{abstract}
This paper describes a universal algorithm for the calculation of the longitudinal radiative interaction force in the electron bunch with a line-charge distribution moving along an arbitrary small-angle trajectory. Practically important case of the bunch moving in an undulator (a wiggler) is studied in detail. Explicit analytical solutions for the radiative interaction force are obtained for a rectangular and for a Gaussian bunch shape. It is shown that the rate of the bunch energy loss due to the radiative interaction forces is equal to the power of coherent radiation in far zone.

The numerical estimations presented in the paper show that the effect of radiative interaction can be important in undulators which are used for generation of coherent infrared radiation.
\end{abstract}




\section{Introduction}

The theory of the radiative interaction of electrons in an intensive microbunch traversing curved trajectory is intensively developed nowadays. This is explained by the practical importance of the radiative effects for beam dynamics in linear colliders $[1,2]$ and shortwavelength free electron lasers $[1,3,4]$. When intensive electron bunch passes bending magnets, bunch compressors, wigglers etc, radiative interaction induces the energy spread in the electron beam and can lead to the transverse emittance dilution in highly dispersive sections of bunch compressors $[5,6]$.

Most of the previous theoretical studies of the radiative interaction (see, e.g., refs. $[7,5,8]$ ) have been focused on the periodical circular motion of a bunch. Recently, the problem connected with transients in a bending magnet of finite length has been considered $[9,10]$. From practical point of view it is important to calculate radiative effects in a more complicated systems such as a sequence of bending magnets, an undulator (a wiggler), etc. In this paper (in Section 2) we present a universal algorithm for the calculation of the radiative interaction of the particles in a line-charge bunch moving on an arbitrary curved small-angle trajectory. This algorithm can be used also to study the radiative effects in a short electron bunch moving along any (not small-angle) trajectory.

In Section 3 we use this general algorithm to find analytical solution for a bunch moving in an undulator (a. wiggler). The solution is obtained using approximations of neglecting transient effects (when bunch enters and leaves an undulator) and neglecting shielding effects (influence of a vacuum chamber on the radiative process). The obtained solution includes periodical oscillations of the radiative force and is written for an arbitrary line density of an electron bunch and an arbitrary value of the undulator parameter. In Sections 4 and 5 the solutions, averaged over period, are obtained in the form of explicit functions for a rectangular and a Gaussian bunches. These results can be used for quick estimations of the considered effect and for testing numerical simulation codes. In Section 6 the applicability region of the considered model is discussed and numerical examples are presented.

\section{Method for calculation}

In this Section we present a universal algorithm for the calculation of the longitudinal (along a particle's velocity) radiative interaction force acting on a particle in an ultrarelativistic electron bunch with a line-charge distribution moving along a plane trajectory. It is assumed that vectors of the velocities are always within a small cone on a part of the trajectory between a position of the bunch head and a retarded position of the tail. Proposed algorithm can be applied, for instance, for any bunch length and small-angle trajectory, or for any trajectory if the bunch is short enough.

The motion of the particles is supposed to be given and we solve only electrodynamic problem. First, we consider the interaction between two particles via longitudinal Lienard- 


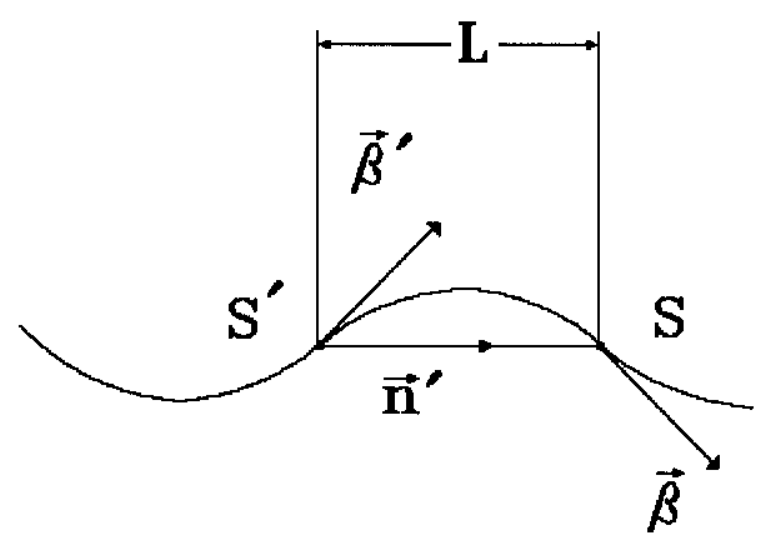

Fig. 1. Interaction of two particles moving on a curved trajectory.

Wiechert electric field and renormalize this interaction in order to exclude from consideration the non-dissipative divergent terms. Second, we obtain the expression for the radiative force acting on a particle within a rectangular bunch. Third, this expression is generalized for a bunch with an arbitrary line density. Finally, the method is illustrated by the consideration of a microbunch moving on a circle.

Let us consider two electrons moving with velocity $\beta c$ (which is close to the velocity of light, $\beta \simeq 1-1 / 2 \gamma^{2}, \gamma^{2} \gg 1$ ) along some small-angle curved trajectory one after another (see Fig.1). The electric field of a back electron produced at source point $S^{\prime}$ at time $t^{\prime}$ reaches a front electron at time $t$ in the point $S$ of the trajectory. Explicit expression for this field is given by Lienard-Wiechert formula [11], containing "Coulomb" and "radiation" terms:

$$
\vec{E}(S)=\vec{E}_{\mathrm{coul}}+\vec{E}_{\mathrm{rad}}=\frac{e}{\gamma^{2}} \frac{\left(\vec{n}^{\prime}-\vec{\beta}^{\prime}\right)}{L^{2}\left(1-\vec{n}^{\prime} \cdot \vec{\beta}^{\prime}\right)^{3}}+\frac{e}{c} \frac{\vec{n}^{\prime} \times\left[\left(\vec{n}^{\prime}-\vec{\beta}^{\prime}\right) \times \dot{\vec{\beta}^{\prime}}\right]}{L\left(1-\vec{n}^{\prime} \cdot \vec{\beta}^{\prime}\right)^{3}}
$$

The distance $L$ between present position of the front electron and retarded position of the back one, the path length $S-S^{\prime}$ between these points along the trajectory, and the distance $s-s^{\prime}$ between the electrons are connected by the retardation condition

$$
S-S^{\prime}-\left(s-s^{\prime}\right)=\beta L
$$

Kinetic energy of the front electron changes under the action of the electric field produced by the back electron:

$$
\frac{d \mathcal{E}}{c d t}=e \vec{\beta} \cdot\left(\vec{E}_{\mathrm{coul}}+\vec{E}_{\mathrm{rad}}\right)
$$

The rate of the energy change of the back electron under the action of the front one is 
given by the expression (in the small-angle approximation):

$$
\frac{d \mathcal{E}^{\prime}}{c d t}=-\frac{e^{2}}{\gamma^{2}\left(s-s^{\prime}\right)^{2}} .
$$

This expression does not depend on a specific shape of a small-angle trajectory and is valid also for the trivial case of a straight-path motion. Both expressions (3) and (4) are divergent at $\left(s-s^{\prime}\right) \rightarrow 0$. We are interested in the study of the curvature effects and remove this divergence subtracting the kinetic energy change which would take place if the electrons were moving on the straight line. This renormalization does not affect the total energy change of the system of two electrons and leads to the following expressions for the "curvature" part (or, radiative part) of the kinetic energy change of the front and of the back electron, respectively:

$$
\begin{aligned}
\frac{d \mathcal{E}_{\mathrm{cur}}}{c d t} & =e \vec{\beta} \cdot \vec{E}_{\mathrm{rad}}+\left[e \vec{\beta} \cdot \vec{E}_{\mathrm{coul}}-\frac{e^{2}}{\gamma^{2}\left(s-s^{\prime}\right)^{2}}\right]=G_{\mathrm{cur}}\left(s-s^{\prime}, S\right), \\
\frac{d \mathcal{E}_{\mathrm{cur}}^{\prime}}{c d t} & =0 .
\end{aligned}
$$

In the following we will refer to the term in square brackets in eq. (5) as to "renormalized Coulomb" term. The back electron does not lose its kinetic energy under the considered interaction, as it is shown by eq. (6).

Let us now study the radiative interaction of the particles in a bunch with a line-charge density $\lambda=$ const. Let a test particle with a coordinate $s$ along the bunch $(s=0$ is the coordinate of the bunch tail) be placed at some point $S$ along the trajectory. The retarded position of the bunch tail is $S_{\mathrm{tr}}$ (see Fig.2). To find the rate of the energy change of the

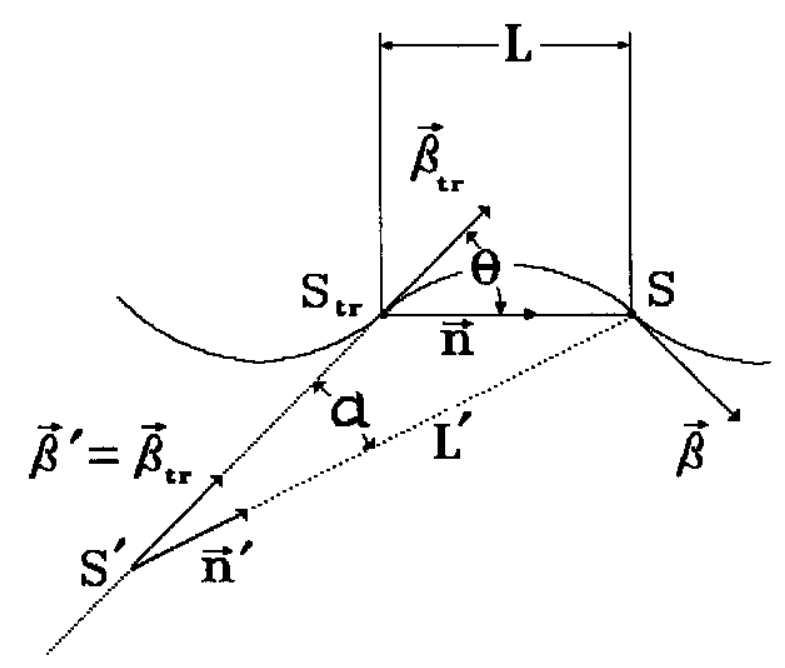

Fig. 2. Illustration of the radiative interaction of particles in a rectangular bunch, including semi-infinite complementary contour. 
test particle we should sum up the action of all the particles behind it. When the particles density is high enough, we can replace this summation by the integration:

$$
\frac{d \mathcal{E}_{\mathrm{cur}}}{c d t}=\lambda \int_{0}^{s} d s^{\prime} G_{\mathrm{cur}}\left(s-s^{\prime}, S\right) \text {. }
$$

Now we can formally add and subtract the action of semi-infinite electron beam with the same linear density:

$$
\frac{d \mathcal{E}_{\text {cur }}}{c d t}=\lambda \int_{-\infty}^{s} d s^{\prime} G_{\mathrm{cur}}\left(s-s^{\prime}, S\right)-\lambda \int_{-\infty}^{0} d s^{\prime} G_{\mathrm{cur}}\left(s-s^{\prime}, S\right)
$$

The first integral in the right hand side of this equation is equal to zero for any small-angle trajectory (see ref. [9] for more detail). It means that the radiative force is equal to zero at any point of infinitely long circuit of dc current. Fields produced by such a circuit are static $^{\mathbf{l}}$. Since the result does not depend on a specific shape of such a complementary circuit, we can choose the complementary circuit convenient for the calculation (it may not coincide with the actual trajectory). It is convenient to assume that retarded positions of particles of complementary semi-infinite beam are placed on the straight line tangential to the actual trajectory at the retarded position of the bunch tail $S_{\mathrm{tr}}$ (see Fig. 2). Apparently, the simplification is connected with the fact that acceleration is equal to zero on this circuit. Thus, the integrand contains only "renormalized Coulomb" term:

$$
\frac{d \mathcal{E}_{\mathrm{cur}}}{c d t}=-\lambda \int_{-\infty}^{0} d s^{\prime} G_{\mathrm{cur}}\left(s-s^{\prime}, S\right)=-\lambda \int_{-\infty}^{0} d s^{\prime}\left[e \vec{\beta} \vec{E}_{\mathrm{coul}}-\frac{e^{2}}{\gamma^{2}\left(s-s^{\prime}\right)^{2}}\right]
$$

The second term is integrated easily and one gets

$$
\frac{d \mathcal{E}_{\mathrm{cur}}}{c d t}=\frac{e^{2} \lambda}{\gamma^{2} s}-\frac{e^{2} \lambda}{\gamma^{2}} \int_{-\infty}^{0} d s^{\prime} \frac{\vec{\beta} \cdot\left(\vec{n}^{\prime}-\vec{\beta}^{\prime}\right)}{\left(L^{\prime}\right)^{2}\left(1-\vec{n}^{\prime} \cdot \vec{\beta}^{\prime}\right)^{3}} .
$$

To perform integration in eq. (10), we go over to a retarded position $S^{\prime}$ as an independent variable, and then - to an angle $\alpha$ (see Fig. 2) which changes from 0 to $\theta$. Finally, we obtain the following expression (see also Fig. 3):

$$
\frac{d \mathcal{E}_{\mathrm{cur}}}{c d t}=e^{2} \lambda \Phi(s, S)=e^{2} \lambda\left(\frac{1}{\gamma^{2} s}-\frac{2}{L} \frac{1+\gamma^{2} \phi \theta}{1+\gamma^{2} \theta^{2}}\right)
$$

\footnotetext{
${ }^{1}$ This is valid also for any finite closed circuit (see, for example, ref. [11]) - in this case, of course, one can not neglect the action of the fields produced by particles moving in front of the test particle.
} 
The retardation condition is written as

$$
s=S-S_{\mathrm{tr}}-\beta L
$$

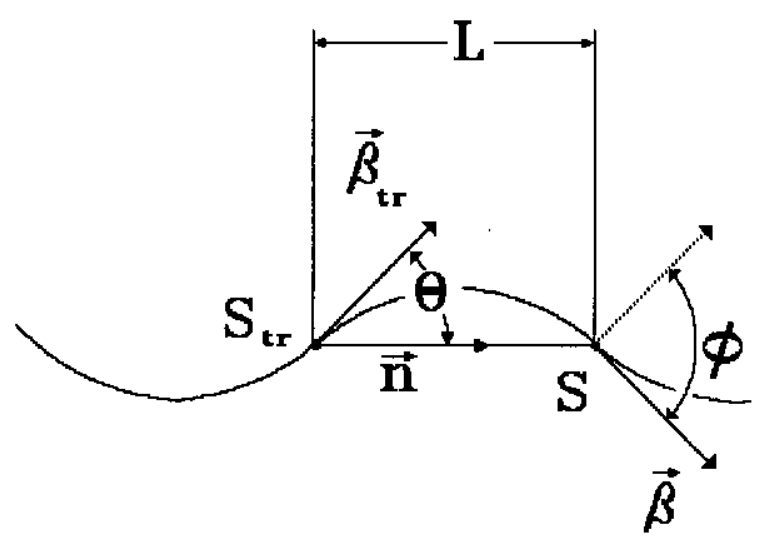

Fig. 3. The scheme for calculation of the radiative force acting on a particle in a rectangular bunch.

We should comment the formulae (11) and (12). Let the test particle be placed at some distance $s$ from the tail of a rectangular bunch. At a given moment of time it passes the point with coordinate $S$ along the trajectory with some definite direction of velocity $\vec{\beta}$ (see Fig. 3). The retarded position of the bunch tail $S_{\text {tr }}$ along the trajectory is defined by the condition (12), where $L$ is the retarded distance. Since we consider a small-angle approximation, one should expand trigonometric functions which will appear in eq. (12), keeping the main term and the next nonvanishing high-order term in angle. At the point $S_{\mathrm{tr}}$ of the trajectory the tail particle has at retarded moment of time the velocity $\vec{\beta}_{\mathrm{tr}}$ and the direction to the present position of the test particle is given by vector $\vec{n}$. The rate of the energy change of the test particle due to the curvature effects is given by formula (11), where $\theta=\arccos \left(\vec{n} \cdot \vec{\beta}_{\mathrm{tr}} / \beta\right)$ and $\phi=\arccos \left(\vec{\beta} \cdot \vec{\beta}_{\mathrm{tr}} / \beta^{2}\right)$. To define the correct signs of these angles, one should use the following rule. If in some polar coordinates the vectors $\vec{\beta}_{\mathrm{tr}}, \vec{n}$, and $\vec{\beta}$ have angles $\psi_{1}, \psi_{2}$ and $\psi_{3}$, respectively, then $\theta=\psi_{1}-\psi_{2}$ and $\phi=\psi_{1}-\psi_{3}$.

Using this algorithm one can calculate the rate of a particle's energy change as a function of its position along a rectangular bunch and of its position along a trajectory (in general case this function may not have explicit form). Then it is easy to generalize the solution for a rectangular bunch with $\lambda(s)=$ const to the case of an arbitrary linear density $\lambda(s)$. One can consider this bunch to be composed of rectangular bunches with a length $\left(s-s^{\prime}\right)$ and a linear density $d s^{\prime}\left[d \lambda\left(s^{\prime}\right) / d s^{\prime}\right]$. Hence,

$$
\frac{d \mathcal{E}_{\mathrm{cur}}}{c d t}=e^{2} \int_{-\infty}^{s} d s^{\prime} \Phi\left(s-s^{\prime}, S\right) \frac{d \lambda\left(s^{\prime}\right)}{d s^{\prime}}
$$

where function $\Phi$ is defined by eq. (11).

Now let us illustrate this algorithm with the simplest case of a microbunch motion on 


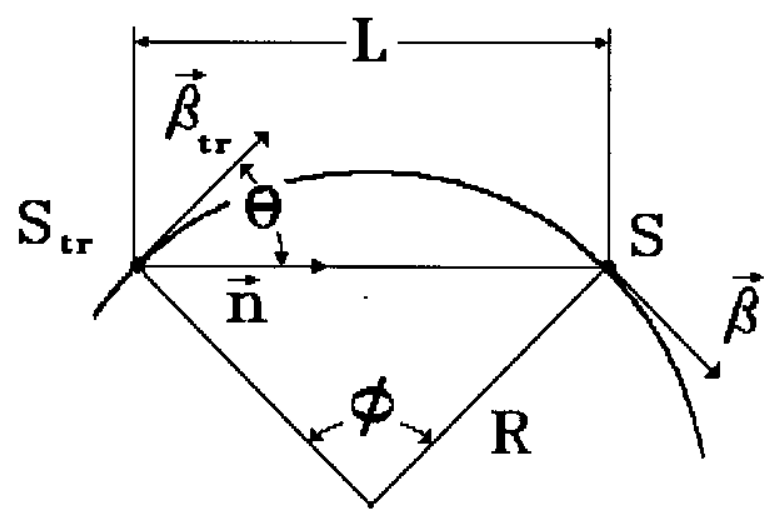

Fig. 4. The scheme for the calculation of the radiative interaction in a rectangular bunch moving on a circle.

a curved trajectory - the periodical circular motion (see Fig. 4). In this geometry $\theta=$ $\phi / 2 \ll 1$ and one easily obtains that the retardation condition (12) is approximated by

$$
s=\frac{R \phi}{2 \gamma^{2}}+\frac{R \phi^{3}}{24}
$$

and expression (11) takes the form [9]:

$$
\frac{d \mathcal{E}_{\mathrm{cur}}}{c d t}=e^{2} \lambda\left(\frac{1}{\gamma^{2} s}-\frac{2}{R \phi} \frac{1+\frac{\gamma^{2} \phi^{2}}{2}}{1+\frac{\gamma^{2} \phi^{2}}{4}}\right)=-\frac{4 e^{2} \lambda \gamma}{R} \frac{(\gamma \phi)\left(8+\gamma^{2} \phi^{2}\right)}{\left(4+\gamma^{2} \phi^{2}\right)\left(12+\gamma^{2} \phi^{2}\right)}
$$

At $\gamma^{2} \phi^{2} \gg 1$ eq. (14) takes the simplified form $s \simeq R \phi^{3} / 24$ and eq. (15) is reduced to the well-known one $[7,5]$ :

$$
\frac{d \mathcal{E}_{\mathrm{cur}}}{c d t}=e^{2} \lambda \Phi(s)=-\frac{4 e^{2} \lambda}{R \phi}=-\frac{2 e^{2} \lambda}{3^{1 / 3} R^{2 / 3} s^{1 / 3}}
$$

The considered case can be referred to as the steady-state regime when the radiative interaction does not depend on a bunch position along a trajectory. Using expressions (13) and (16), for the case of an arbitrary function of linear density we obtain $[7,5,8]$ :

$$
\frac{d \mathcal{E}_{\mathrm{cur}}}{c d t}=-\frac{2 e^{2}}{3^{1 / 3} R^{2 / 3}} \int_{-\infty}^{s} \frac{d s^{\prime}}{\left(s-s^{\prime}\right)^{1 / 3}} \frac{d \lambda\left(s^{\prime}\right)}{d s^{\prime}}
$$

In the next Section we use the described algorithm for the calculation of the radiative interaction of the electrons in a bunch moving in an undulator. The transient effects (when a bunch enters and leaves an undulator) are not considered in this paper. Nevertheless, we should note that the algorithm described in this Section allows one to calculate these transients, too. The subscript "cur" will not be used in the following Sections and one should keep in mind that we always mean the kinetic energy change due to curvature 
effects. The terms, which are omitted in our consideration, describe trivial longitudinal space-charge forces in a bunch moving on a straight line and can be calculated separately.

\section{General solution for a bunch moving in an undulator}

Let us consider rectangular electron bunch of a length $l_{b}$ with a linear density $\lambda=N / l_{b}$ moving in the $z$ direction through an undulator with magnetic field

$$
H_{x}=H_{w} \cos \left(k_{w} z\right)
$$

The transverse and longitudinal velocities of an electron can be approximated by

$$
\beta_{y}=\frac{K}{\gamma} \sin \left(k_{w} z\right), \quad \beta_{z}=\beta-\frac{K^{2}}{2 \gamma^{2}} \sin ^{2}\left(k_{w} z\right)
$$

where $K=e H_{w} / k_{w} m c^{2}$ is the undulator parameter, $\gamma=\mathcal{E} / m c^{2}$ is relativistic factor, $\left(1+K^{2} / 2\right) / \gamma^{2} \ll 1$ and $\beta \simeq 1-1 / 2 \gamma^{2}$. Transverse coordinate changes as follows:

$$
y=-\frac{K}{\gamma k_{w}} \cos \left(k_{w} z\right)
$$

We suppose the motion of particles to be given and solve only electrodynamic problem. Our goal is to calculate the rate of the energy change of an electron in the bunch due to the radiative interaction force as a function of electron's position $s$ along the bunch ( $s=0$ is the coordinate of the bunch tail) and of its position $z$ along the undulator. The calculations are based on the general approach described in the previous Section. Leaving the details of the calculations, we present here the following result:

$$
\frac{d \mathcal{E}}{c d t}=e^{2} k_{w} \lambda D(\hat{s}, K, \hat{z})
$$

where

$$
\begin{aligned}
& D(\hat{s}, K, \hat{z})=\frac{1}{\hat{s}}-2 \frac{\Delta-K^{2} B(\Delta, \hat{z})[\sin \Delta \cos \hat{z}+(1-\cos \Delta) \sin \hat{z}]}{\Delta^{2}+K^{2} B^{2}(\Delta, \hat{z})}, \\
& B(\Delta, \hat{z})=(1-\cos \Delta-\Delta \sin \Delta) \cos \hat{z}+(\Delta \cos \Delta-\sin \Delta) \sin \hat{z},
\end{aligned}
$$

and $\Delta$ is the solution of the transcendental equation: 


$$
\begin{aligned}
& \hat{s}=\frac{\Delta}{2}\left(1+\frac{K^{2}}{2}\right)+\frac{K^{2}}{4 \Delta}\{[2(1-\cos \Delta)-\Delta \sin \Delta] \\
& \times(\cos \Delta \cos 2 \hat{z}+\sin \Delta \sin 2 \hat{z})-2(1-\cos \Delta)\} .
\end{aligned}
$$

Here the following reduced variables are used: $\hat{s}=\gamma^{2} k_{w} s$ and $\hat{z}=k_{w} z$. To explain the physical sense of variable $\Delta$, let us represent it in the following form: $\Delta=k_{w}\left(z-z_{t_{r}}\right)$. Here $z$ and $z_{t r}$ are projections on the undulator axis of the current position of reference particle and of the retarded position of the bunch tail, respectively.

It follows from geometrical symmetry of the problem and from eqs. (19), (20) and (21) that function $D$ is periodical with respect to the position of reference particle $z$ along the undulator and the period is equal to the half of the undulator period $\pi / k_{w}$ (the period is equal to $\pi$ with respect to the normalized position $\hat{z}$ ).

To check the validity of expression (18) let us consider the limits when this expression has simple physical interpretation. First of all, it is seen that in the limit of $\hat{s} \rightarrow \infty$ function $D$ tends to zero, i.e. at any point of infinitely long bunch the radiative interaction force is equal to zero as it should be.

Now let the bunch be shorter than characteristic wavelength of spectrum of incoherent radiation, which is of the order of $\left(k_{w} \gamma^{2}\right)^{-1}$ for small values of $K$ and of the order of $\left(K k_{w} \gamma^{2}\right)^{-1}$ for large values of $K$. When

$$
\hat{s} \ll 1, \quad \hat{s}^{2} K^{2} \ll 1,
$$

function $D$ is reduced to the following simple form:

$$
D=-\frac{4}{3} K^{2} \hat{s} \cos ^{2} \hat{z}
$$

The rate of the energy loss by the whole bunch can be calculated as follows:

$$
\frac{d \mathcal{E}_{b}}{c d t}=\int_{0}^{l_{b}} d s \lambda \frac{d \mathcal{E}}{c d t} .
$$

Substituting expressions (18) and (23) in eq. (24) we obtain:

$$
\frac{d \mathcal{E}_{b}}{c d t}=-\frac{2}{3}\left[N e K k_{w} \gamma \cos \left(k_{w} z\right)\right]^{2}
$$

Using the definition of the parameter $K$ we can rewrite this expression in the following form:

$$
\frac{d \mathcal{E}_{b}}{c d t}=-\frac{2}{3} N^{2} r_{e}^{2} \gamma^{2} H_{w}^{2} \cos ^{2}\left(k_{w} z\right)
$$


where $r_{e}=e^{2} / m c^{2}$ is the classical radius of electron. One can see that in the limit of a "short" bunch the energy loss is identical to that of a single particle with the charge $\mathrm{Ne}$. So, in this limit formula (18) provides correct and physically transparent result. This indicates also that renormalization procedure (5) has been performed correctly. Indeed, result (25) could not be obtained without taking into account the "renormalized Coulomb" term.

Now let us consider the limit of $\Delta \ll 1$. In this case the result should depend on the value of the local magnetic field only (or, on local curvature), and solution (18) must be reduced to that corresponding to a circular motion. The approximate form of eq. (21) can be written as follows:

$$
\hat{s} \simeq \frac{\Delta}{2}+\frac{K^{2}}{24} \Delta^{3} \cos ^{2} \hat{z}
$$

The first term in the right hand side of this equation comes from the difference between the electron's velocity and the velocity of light, and the second one is due to a local curvature of the trajectory. As a result, function $D$ takes the form:

$$
D=-\frac{4 \Delta K^{2} \cos ^{2} \hat{z} \quad\left(8+\Delta^{2} K^{2} \cos ^{2} \hat{z}\right)}{\left(4+\Delta^{2} K^{2} \cos ^{2} \hat{z}\right)\left(12+\Delta^{2} K^{2} \cos ^{2} \hat{z}\right)}
$$

Taking into account that

$$
\frac{k_{w}^{2} K^{2} \cos ^{2} \hat{z}}{\gamma^{2}}=\frac{1}{R^{2}}
$$

where $R$ is a local radius of curvature, we can obtain that the solution (18), (26) and (27) is identical to (15) and (14). When conditions (22) are satisfied, expression (27) takes the simple form (23). Solution (27) is reduced to (16) at $(K|\cos \hat{z}|)^{-1} \ll \Delta \ll 1$. The latter asymptote means that the bunch is much shorter than the wavelength of the first harmonic of the undulator radiation but is much longer than the characteristic wavelength $R / \gamma^{3}$ of the synchrotron radiation spectrum (which is radiated by a single electron at large values of the parameter $K$ ).

We have checked that solution (18) for a rectangular bunch has correct asymptotical behavior. Now we can generalize this solution for the case of a bunch with an arbitrary linear density $\lambda(s)$. As it was mentioned in the previous Section, this general solution is the convolution of the solution for a rectangular bunch with the derivative of a linear density function:

$$
\frac{d \mathcal{E}}{c d t}=e^{2} k_{w} \int_{-\infty}^{s} d s^{\prime} D\left(\hat{s}-\hat{s}^{\prime}, K, \hat{z}\right) \frac{d \lambda\left(s^{\prime}\right)}{d s^{\prime}}
$$


In the next Sections we will study the rate of a particle's energy change averaged over $z$ :

$$
\frac{d \overline{\mathcal{E}}}{c d t}=e^{2} k_{w} \int_{-\infty}^{s} d s^{\prime} \bar{D}\left(\hat{s}-\hat{s}^{\prime}, K\right) \frac{d \lambda\left(s^{\prime}\right)}{d s^{\prime}}
$$

where

$$
\bar{D}(\hat{s}, K)=\frac{1}{\pi} \int_{0}^{\pi} d \hat{z} D(\hat{s}, K, \hat{z}) .
$$

\section{Averaged solution for a rectangular bunch}

In this Section we study the averaged solution (29) and (30) for the particular case of a rectangular bunch of a length $l_{b}$ with a linear density $\lambda=N / l_{b}$. Let us rewrite this solution in the following form:

$$
\frac{d \overline{\mathcal{E}}}{c d t}=\frac{e^{2} k_{w} N}{l_{b}} \tilde{D}(\hat{s}, K)
$$

The averaged function $\bar{D}(\hat{s}, K)$ is calculated using the eqs. (30) and (19) - (21). For small values of the undulator parameter, $K \ll 1$, we have

$$
\bar{D}(\hat{s}, K)=-K^{2}\left(\frac{\sin ^{2} \hat{s}}{\hat{s}}+\frac{\sin 2 \hat{s}}{2 \hat{s}^{2}}-\frac{\sin ^{2} \hat{s}}{\hat{s}^{3}}\right)
$$

It is easy to see that at small values of $\hat{s}$ this expression is reduced to that given by eq. (23) averaged over the $z$ coordinate. Let us note that the first term in the brackets of eq. (32) takes its origin from the "radiation" term in eq. (5), but the second and third ones come from the "renormalized Coulomb" term.

The energy losses by the whole bunch are given by:

$$
\frac{d \overline{\mathcal{E}}_{\mathrm{b}}}{c d t}=\frac{e^{2} N^{2}}{\gamma^{2} l_{\mathrm{b}}^{2}} \int_{0}^{\hat{l}_{\mathrm{b}}} \bar{D}(\hat{s}, K) d \hat{s}
$$

where

$$
\hat{l}_{\mathrm{b}}=\gamma^{2} k_{\mathrm{w}} l_{\mathrm{b}}
$$


Performing integration, we obtain:

$$
\frac{d \overline{\mathcal{E}}_{\mathrm{b}}}{d t}=-\frac{c e^{2} N^{2} K^{2}}{2 \gamma^{2} l_{\mathrm{b}}^{2}}\left[\ln \left(2 \hat{l}_{\mathrm{b}}\right)-\operatorname{Ci}\left(2 \hat{l}_{\mathrm{b}}\right)+C+\left(\frac{1-\cos \left(2 \hat{l}_{\mathrm{b}}\right)}{2 \hat{l}_{\mathrm{b}}^{2}}-1\right)\right],
$$

where $C=0.577 \ldots$ is Euler's constant and $\operatorname{Ci}(\ldots)$ is integral cosine [12].

Let us show that the rate of the energy losses by the electron bunch is equal to the power of coherent radiation in the far diffraction zone. The power of coherent radiation in far zone is calculated as the integral of the power spectral density

$$
\frac{d P_{c o h}}{d \omega}=N^{2} \eta(\omega) \frac{d P}{d \omega}
$$

where $\eta(\omega)$ is a bunch form factor (squared module of the Fourier transform of a bunch shape). The form factor for the rectangular bunch of the length $l_{b}$ is:

$$
\eta(\omega)=\left(\sin \frac{\omega l_{b}}{2 c}\right)^{2}\left(\frac{\omega l_{b}}{2 c}\right)^{-2}
$$

Function $d P / d \omega$ entering eq. (35) is the spectral density of the radiation power emitted by a single electron. For the first time it has been calculated in ref. [13]. In the case under consideration (small value of the undulator parameter $K$ ), this function is reduced to the following simple form:

$$
\frac{d P}{d \omega}=\frac{e^{2} K^{2} \omega}{4 c \gamma^{2}}\left(1-\frac{\omega}{c k_{w} \gamma^{2}}+\frac{\omega^{2}}{2 c^{2} k_{w}^{2} \gamma^{4}}\right)
$$

where $\omega$ changes from zero to $2 c k_{w} \gamma^{2}$. Substituting functions (36) and (37) in eq. (35) and integrating within these limits, one obtains that $P_{\text {coh }}$ exactly coincides with $d \overline{\mathcal{E}}_{\mathrm{b}} / d t$ taken with the opposite sign.

We should note that the "renormalized Coulomb" contribution to the total power, being placed in the brackets (...) in eq. (34), is not negligible even for a long bunch, $\hat{l}_{b} \gg 1$. In this case the contribution of the main ("radiation") term is only logarithmically larger. This shows the importance of "Coulomb" part of the Lienard-Wiechert field in the radiative interaction processes.

We have considered the case of small values of the parameter $K$. In the case of an arbitrary value of this parameter function $\bar{D}(\hat{s}, K)$ takes much more complicated form than eq. (32). We present here the asymptotical behaviour of this general averaged solution for a rectangular bunch at large distances from the tail $\left(\hat{s} /\left(1+K^{2} / 2\right) \gg 1\right)$ :

$$
\bar{D}(\hat{s}, K) \simeq-\frac{\left(1+K^{2} / 2\right)}{\hat{s}}\left[2\left(1-\frac{1}{\sqrt{1+K^{2}}}\right) \sin ^{2}\left(\frac{\hat{s}}{1+K^{2} / 2}\right)\right.
$$




$$
\left.+\frac{1}{\sqrt{1+K^{2}}}-\frac{1}{1+K^{2} / 2}\right] \text {. }
$$

At small values of $K$ this solution is reduced to the first term in eq. (32).

\section{Averaged solution for a Gaussian bunch}

In this Section we consider a bunch with a Gaussian distribution of linear density:

$$
\lambda(s)=\frac{N}{\sqrt{2 \pi} \sigma} \exp \left[-\frac{s^{2}}{2 \sigma^{2}}\right] .
$$

Let us introduce the bunch length parameter

$$
p=\frac{\gamma^{2} k_{w} \sigma}{1+K^{2} / 2}
$$

The averaged solution for the Gaussian bunch can be written in the form:

$$
\frac{d \overline{\mathcal{E}}}{c d t}=\frac{e^{2} N K^{2}}{\sqrt{2 \pi} \sigma^{2} \gamma^{2}} \bar{G}(p, K, x),
$$

where $x=s / \sigma$.

In general case one can calculate function $\bar{G}$ numerically using the eq. (29). Here we study practically important case of a long bunch, $p \gg 1$. As in the previous Section, we start with the limit of small $K$. Under these conditions function $\bar{G}$ can be calculated using eqs. (29) and (32):

$$
\bar{G}(p, x)=\frac{x}{2} \exp \left(-\frac{x^{2}}{2}\right) \ln p+F(x) .
$$

Here parameter $p$ is reduced to $p \simeq \gamma^{2} k_{w} \sigma$, and function $F(x)$ has the form:

$$
\begin{aligned}
F(x)= & \frac{1}{4}(C+3 \ln 2-2) x \exp \left(-\frac{x^{2}}{2}\right)-\sqrt{\frac{\pi}{8}}\left[1+\operatorname{erf}\left(\frac{x}{\sqrt{2}}\right)\right. \\
& \left.-x \exp \left(-\frac{x^{2}}{2}\right) \int_{0}^{x} d x^{\prime} \exp \left(\frac{\left(x^{\prime}\right)^{2}}{2}\right)\left(1+\operatorname{erf}\left(\frac{x^{\prime}}{\sqrt{2}}\right)\right)\right],
\end{aligned}
$$

where $\operatorname{erf}(\ldots)$ is the error function [12]. The plot of function $F(x)$ is presented in Fig. 5 and Fig. 6 presents the plots of function $\bar{G}$ calculated at different values of parameter $p$. 


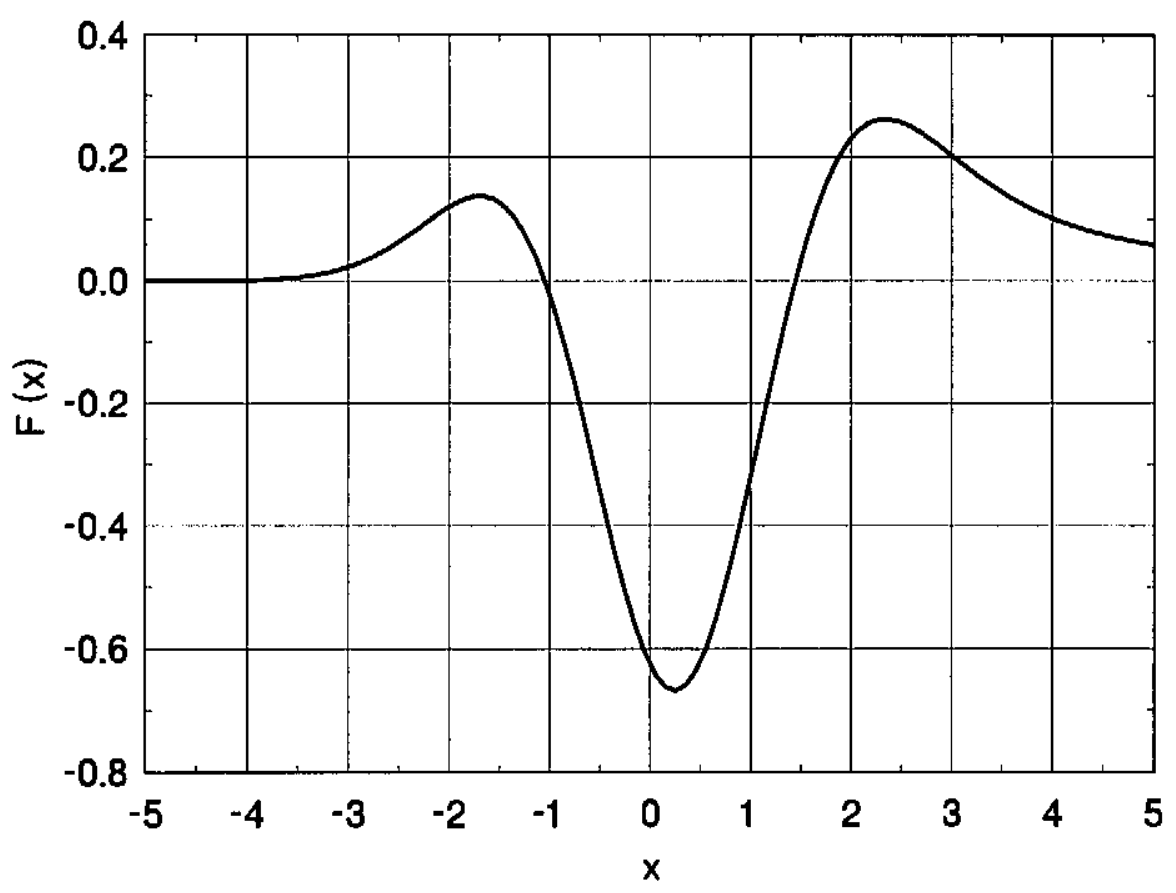

Fig. 5. Function $F(x)$ given by eq. (42).

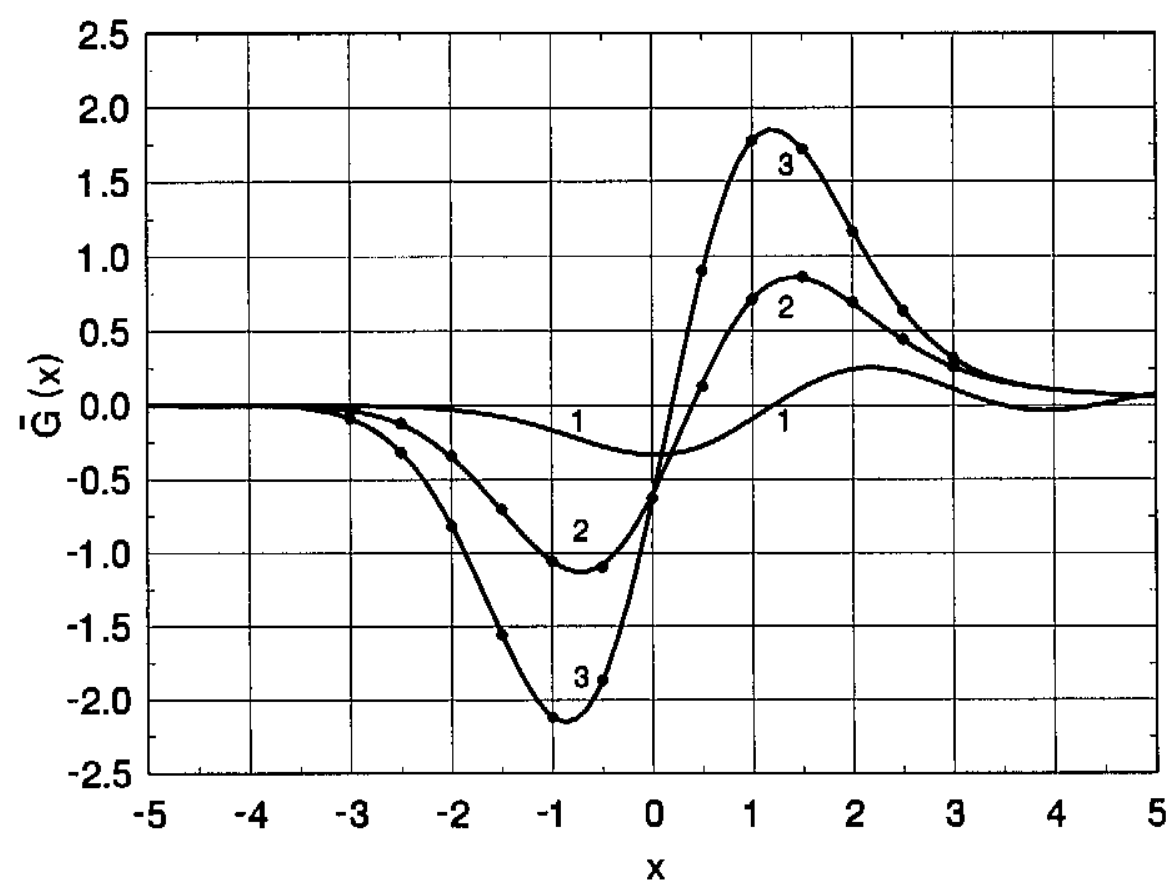

Fig. 6. Function $\bar{G}$ for small value of the undulator parameter $\mathrm{K}$ and different values of the bunch length parameter $p$. Curve (1): $p=1$, curve (2):p=30, and curve (3):p=1000. The curves are the results of numerical integration of eq. (29) and the circles are calculated with the help of analytical formula (41) for large values of parameter $p$. 
In the case of an arbitrary value of the undulator parameter $K$, it is difficult to find explicit analytical solution. Nevertheless, using the results of numerical integration of eq. (29), we can write function $\vec{G}$ in the following form $(p \gg 1)$ :

$$
\bar{G}(p, K, x)=\frac{x}{2} \exp \left(-\frac{x^{2}}{2}\right)[\ln p+g(K)]+F(x),
$$

where function $g(K)$ changes from 0 to 1 when $K$ changes from small to large values. The plot of this function is presented in Fig. 7 .

We also present here the solution for a short bunch, $p \ll 1$, and an arbitrary value of parameter $K$ :

$$
\bar{G}(p, K, x)=\frac{\sqrt{2 \pi}}{3} p^{2}\left(1+\frac{K^{2}}{2}\right)^{2}\left[1+\operatorname{erf}\left(\frac{x}{\sqrt{2}}\right)\right] .
$$

The solution for a long bunch (43) is the most important one from practical point of view. For this expression to be valid, the rate of the energy losses by the whole bunch must be equal to the power of coherent radiation in far zone. First, we should calculate the rate of the energy loss by the whole bunch. Multiplying expression (40) (where the function $\vec{G}$ is

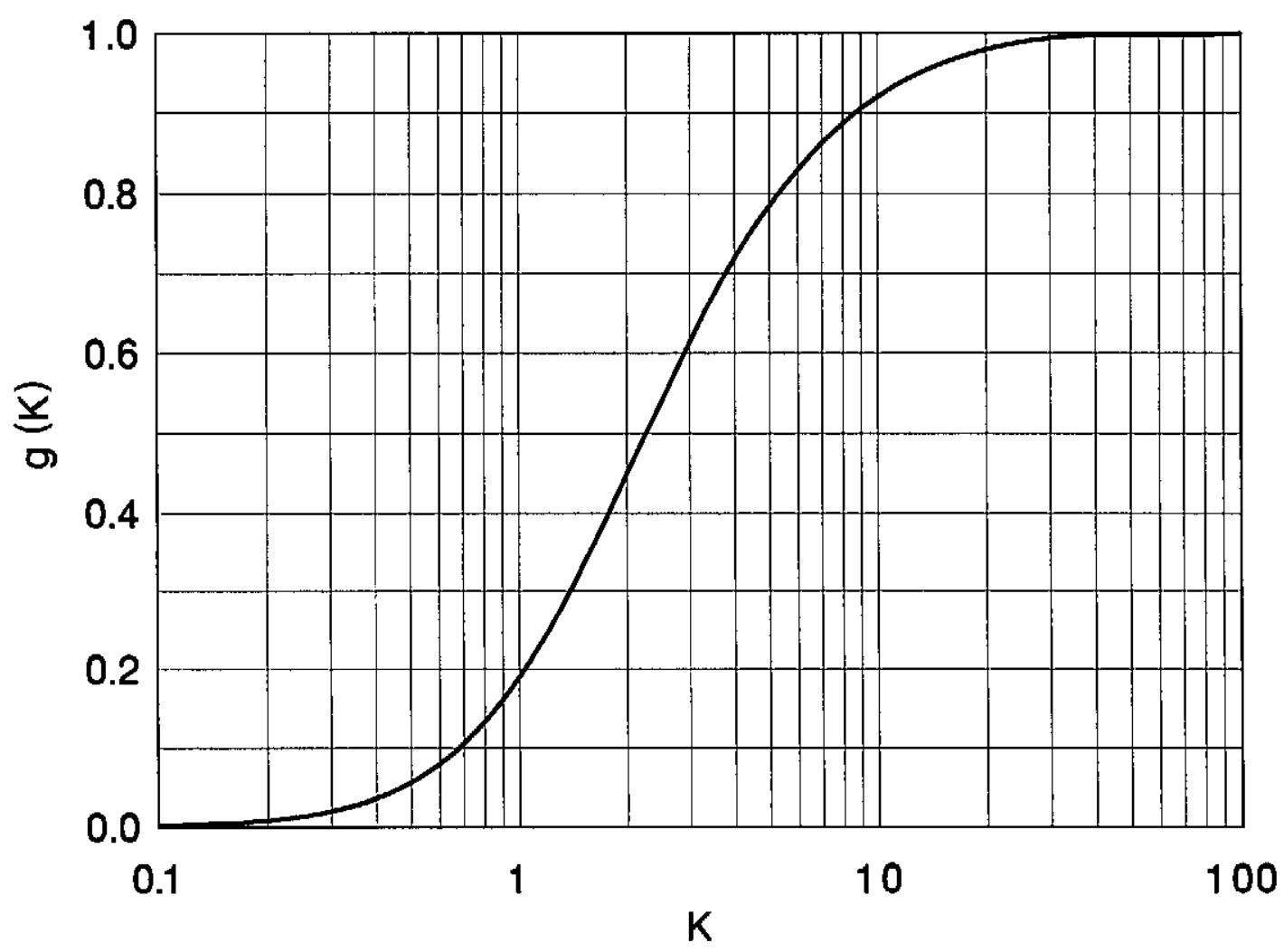

Fig. 7. Function $g(K)$ entering eq. (43). 
defined by eq. (43)) by the function of linear density (39) and performing the integration, we get:

$$
\frac{d \overline{\mathcal{E}}_{\mathrm{b}}}{d t}=-\frac{c e^{2} N^{2} K^{2}}{8 \sigma^{2} \gamma^{2}}
$$

Second, we use formula (35) to calculate the radiation power in far zone. The form factor of the bunch is given by

$$
\eta(\omega)=\exp \left(-\frac{\sigma^{2} \omega^{2}}{c^{2}}\right)
$$

It is seen that typical frequencies of coherent radiation are below $c / \sigma$. This means that in the case of a long bunch, $p \gg 1$, we can use the asymptotical formula for power spectral density of radiation by a single electron [13] , assuming the frequency to be much less than the frequency of the first harmonic of the undulator radiation $\left(\omega \ll c k_{w} \gamma^{2} /\left(1+K^{2} / 2\right)\right)$ :

$$
\frac{d P}{d \omega} \simeq \frac{e^{2} K^{2} \omega}{4 c \gamma^{2}}
$$

Substituting eqs. (46) and (47) into eq. (35) and performing the integration, we obtain that the coherent radiation power in far zone exactly coincides with the bunch power losses (45) taken with the opposite sign.

Finally, let us present the formula for the induced correlated energy spread in the Gaussian bunch due to the radiative interaction. Using expression (43) we write down this formula in the form convenient for practical calculations $(p \gg 1)$ :

$$
\frac{d \sigma_{\gamma}}{c d t}=0.219 \frac{I K^{2}}{I_{\mathrm{A}} \sigma \gamma^{2}} \sqrt{[\ln p+g(K)]^{2}+0.933[\ln p+g(K)]-0.786}
$$

where $I=N e c / \sqrt{2 \pi} \sigma$ is the peak current, $I_{A}=17 \mathrm{kA}$ is Alfven current, and

$$
m c^{2} \sigma_{\gamma}=\sqrt{\left\langle\overline{\mathcal{E}}^{2}\right\rangle-\langle\overline{\mathcal{E}}\rangle^{2}}
$$

\section{Discussion}

Let us discuss the applicability region of the results obtained in the paper. The model approximations are using of the model of the line-charge bunch and neglecting the influence of the vacuum chamber on the process of radiative interaction. Besides, we have considered the steady-state regime, i.e. transients in an undulator of a finite length have been excluded out of consideration. Of course, the most correct way to set applicability conditions of some theoretical model is the consideration of more general model, which 
includes all the effects neglected in the simple model. The consideration of more general model is under way. Here we will try to make some rough estimations of applicability conditions of the simple model considered in this paper.

We can perform such estimations for the practically important case of a long bunch, $\sigma \gg\left(1+K^{2} / 2\right) / \gamma^{2} k_{w}$. First, we consider the transient effects. If one is not interested in the behaviour of the radiative forces after the bunch leaves the undulator (which is true for FELs), then the only transient when the bunch enters the undulator is of interest. For such a case we can write down the following limitation on the undulator (the wiggler) length $L_{w}$, allowing us to neglect transient effects:

$$
L_{w} \gg \sigma \gamma_{z}^{2}
$$

where $\sigma \gamma_{z}^{2}=\sigma \gamma^{2} /\left(1+K^{2} / 2\right)$ is typical radiation formation length.

Second, we estimate the region where we can neglect the influence of the bunch transverse size and of the vacuum pipe. It follows from simple geometrical consideration that a characteristic measure distinguishing these effects is the mean geometric value of the bunch length and the radiation formation length. Thus, we can roughly estimate the region where the considered effects can be neglected:

$$
\sigma_{\perp} \ll \sigma \gamma_{z} \ll b
$$

Here $\sigma_{\perp}$ and $b$ are transverse sizes of the bunch and of the vacuum chamber, respectively.

We should note that when the above mentioned limitations are not satisfied and the considered effects become to be important, they will lead to a suppression of the radiative interaction. In other words, the model considered in this paper means the worst-case approximation, which makes it useful for quick estimations of the radiative interaction effects. Also, numerical simulation codes can be checked by taking them to the corresponding extreme case and comparing them with analytical results of this paper.

Let us consider two numerical examples. The first one is $6 \mathrm{~nm}$ SASE FEL being under construction at the TESLA Test Facility at DESY [4]. The energy is $1 \mathrm{GeV}$, the rms bunch length is $50 \mu \mathrm{m}$, the peak current is $2.5 \mathrm{kA}$, the undulator period is $2.73 \mathrm{~cm}, K$ is 1.27 and the undulator length is $27 \mathrm{~m}$. Substituting these numbers into formula (48), we obtain that $\sigma_{\gamma} / \gamma=4 \times 10^{-5}$ which is negligible. Besides, the condition (49) and the condition (50) for shielding (the diameter of vacuum chamber is $1 \mathrm{~cm}$ ) are not satisfied. This will lead to further reduction of the effect. We should note that such a situation is typical for the projects of VUV and X-ray FELs.

The second example is SUNSHINE experiment [14] with coherent infrared radiation produced by sub-picosecond electron bunches. The energy is $16.5 \mathrm{MeV}$, the bunch length is $240 \mu \mathrm{m}$, the peak current is $280 \mathrm{~A}$, the undulator period is $7.7 \mathrm{~cm}, K$ varies from 0.3 to 3 and the undulator length is $2 \mathrm{~m}$. The model presented in this paper describes well this region of parameters. The estimation of the effect for $K=2$ yields $\sigma_{\gamma} / \gamma \simeq 10^{-2}$ which 
could significantly influence on longitudinal beam dynamics. In particular, a transformation of the energy modulation to the density modulation may lead to a nonlinear growth of the coherent emission with the undulator length that was observed in this experiment. Based on the general formulae presented in Section 3, one can derive the approximate algorithm for a fast numerical solution of the self-consistent problem: longitudinal phase space distribution changes due to the radiative interaction and the latter is defined by former.

Finally, we should note that the results of this paper can be useful for the design of wigglers as bunch compressors for linear colliders $[2,15]$. 


\section{References}

[1] "Conceptual Design of a $500 \mathrm{GeV} e^{+} e^{-}$Linear Collider with Integrated X-ray Laser Facility", DESY 1997-048, ECFA 1997-182.

[2] "Zeroth-Order Design Report for the Next Linear Collider", LBNL-PUB-5424, SLAC Report 474, UCRL-ID-124161 (May 1996).

[3] R. Tatchyn et al., Nucl. Instr. and Methods A375(1996)274.

[4] J. Rossbach, Nucl. Instr. and Methods A375(1996)269.

[5] Ya.S. Derbenev, J. Rossbach, E.L. Saldin, V.D. Shiltsev, "Microbunch Radiative Tail-Head Interaction", DESY Print, TESLA-FEL 95-05, Hamburg(1995).

[6] M. Dohlus and T. Limberg, Nucl. Instr. and Methods A393(1997)494.

[7] L.V. Iogansen and M.S. Rabinovich, Sov. Phys. JETP, vol.37(10), 1960, p.83.

[8] J.B. Murphy, S. Krinsky, and R.L. Glukstern, Particle Accelerators 57(1997)9.

[9] E.L. Saldin, E.A. Schneidmiller, and M.V. Yurkov, "On the Coherent Radiation of an Electron Bunch Moving in an Arc of a Circle", DESY print TESLA-FEL 96-14 (November 1996); Nucl. Instr. and Methods, in press.

[10] R. Li, C.L. Bohn, and J.J. Bisognano, "Shielded transient self-interaction of a bunch entering a circle from a straight path", presented at SPIE's International Symposium on Optical Science, Engineering, and Instrumentation (San-Diego, CA, July 1997).

[11] J.D. Jackson, Classical Electrodynamics, (Wiley, New York, 1975).

[12] M. Abramowitz and I.A. Stegun, Handbook of Mathematical Functions, (National Bureau of Standards, 1964).

[13] D.F. Alferov, Yu.A. Bashmakov, and E.G. Bessonov, Sov. Phys. Tech. Phys. 18(1974)1336.

[14] H. Wiedemann, "Observation of Coherent Radiation from Sub-picosecond Electron Bunches", Proc. of the 10th Advanced ICFA Beam Dynamics Workshop on 4th Generation Light Sources (Grenoble, January 1996).

[15] T.O. Raubenheimer, P. Emma, S. Kheifets, "Chicane and Wiggler Based Bunch Compressors for Future Linear Colliders", Proc. of the 1993 Particle Accelerator Conference (Washington, D.C., 1993) and SLAC-PUB-6119. 